\title{
Multifocal Diffuse Sclerosing Papillary Thyroid Carcinoma: A Rare Variant with Diagnostic Challenges
}

\author{
Dr. Ann Maria Sunny ${ }^{1 *}$, Dr. Sankar $S^{2}$ \\ ${ }^{1}$ Junior Resident, ${ }^{2}$ Professor and HOD, Department of Pathology Government Medical College, Kottayam, Kerala, India
}

DOI: $10.36348 /$ sjpm.2022.v07i01.003 $\quad$ | Received: 12.12.2021 | Accepted: 13.01 .2022 | Published: 24.01 .2022

*Corresponding author: Dr. Ann Maria Sunny

Junior Resident Department of Pathology Government Medical College Kottayam

\section{Abstract}

Diffuse sclerosing papillary thyroid carcinoma (DS PTC) is a relatively rare variant of papillary thyroid carcinoma. It is characterized by distinct histological features like scattered microscopic tumor islands, diffuse sclerosis, lymphocytic infiltration, numerous psammoma bodies, radiological features like snowstorm appearance and biologically aggressive behavior in the form of invasion and metastasis. A preoperative diagnosis is often challenging in the absence of nodules and scanty fine needle aspiration cytology sample. We describe a case of multifocal diffuse sclerosing variant of papillary thyroid carcinoma in a 29 year old young male who presented with a swelling in the right side of neck of 2 month duration in the absence of a visible thyroid swelling. Neck swelling was non tender, well defined, firm, not moving with deglutition. Laboratory studies showed normal thyroid function, neck ultrasound revealed heteroechoic right lobe with multiple punctate echogenic foci and enlarged right sided cervical nodes with echogenic foci, fine needle aspiration cytology of which was consistent with metastasis from papillary adenocarcinoma. Based on the above investigations, a total thyroidectomy with central compartment lymph node dissection and modified radical neck dissection of right side was performed. Histological analysis revealed Multifocal Diffuse Sclerosing variant of papillary Thyroid Carcinoma. Clinical and histological challenges usually occur when DS PTC present as diffuse thyroid enlargement, dispersed microscopic tumor islands, extensive fibrosis, abundant lymphocytic infiltration mimicking thyroiditis and when the fine needle aspiration cytology sample is non representative.

Key words: Diffuse sclerosing variant, papillary thyroid carcinoma, Hashimoto's thyroiditis, ultrasound, diagnostic challenges.

Copyright (C) 2022 The Author(s): This is an open-access article distributed under the terms of the Creative Commons Attribution 4.0 International License (CC BY-NC 4.0) which permits unrestricted use, distribution, and reproduction in any medium for non-commercial use provided the original author and source are credited.

\section{INTRODUCTION}

Papillary carcinoma is the most common malignant neoplasm of thyroid gland. Diffuse sclerosing variant papillary thyroid carcinoma (DSV PTC) - a rare histological variant of papillary thyroid carcinoma, originally described by Vickery et al. in 1985 and was recognized as a morphological variant of PTC by WHO in 1988 is recognized as biologically aggressive [1]. It occurs at younger age group with a female preponderance and has high incidence of metastasis than conventional PTC. It is characterized by diffuse involvement of one or both thyroid lobe with prominent squamous metaplasia, numerous psammoma bodies and dense sclerosis with a background of lymphocytic infiltration [2]. Because DSV PTC always permeates the entire gland which cause diffuse thyroid enlargement without a dominant nodule and because histological analysis show copious inflammation and scattered microscopic tumor islands, the pre-operative diagnosis of DSV PTC is challenging [3].

\section{CASE REPORT}

Our case, who was a 29 year old young male presented with a swelling on the right side of neck of 2 month duration. The swelling was painless without any change in size. The patient did not have any unusual palpitation, body tremor, loss of weight, loss of appetite, and difficulty in swallowing, change in voice. The patient had no family history of thyroid disease, nor a personal history related to a preexisting thyroid pathologic process or previous irradiation to neck region. 
Physical examination revealed a 5 x $5 \mathrm{~cm}$ firm, non-tender swelling with nodular surface in the right side of neck at the medial margin of sternocleidomastoid muscle and is extending upto posterior triangle lateral to lateral margin of sternocleidomastoid. The swelling is not moving with deglutition. There was no midline swelling. Ultrasound of neck revealed heteroechoic right lobe with multiple punctate echogenic foci, heteroechoic left lobe and isthmus with increased vascularity and echogenic foci in enlarged right cervical lymph nodes. Serological analysis showed normal Thyroid function. Fine needle aspiration cytology of right sided neck swelling showed atypical epithelial cells arranged in papillaroid clusters with cells having moderate eosinophilic cytoplasm, round-oval nucleus with few showing prominent intranuclear inclusion in a background of scattered lymphocytes. Pap smear was consistent with metastasis from papillary adenocarcinoma. Since intranuclear inclusions were seen, a primary in thyroid was likely.

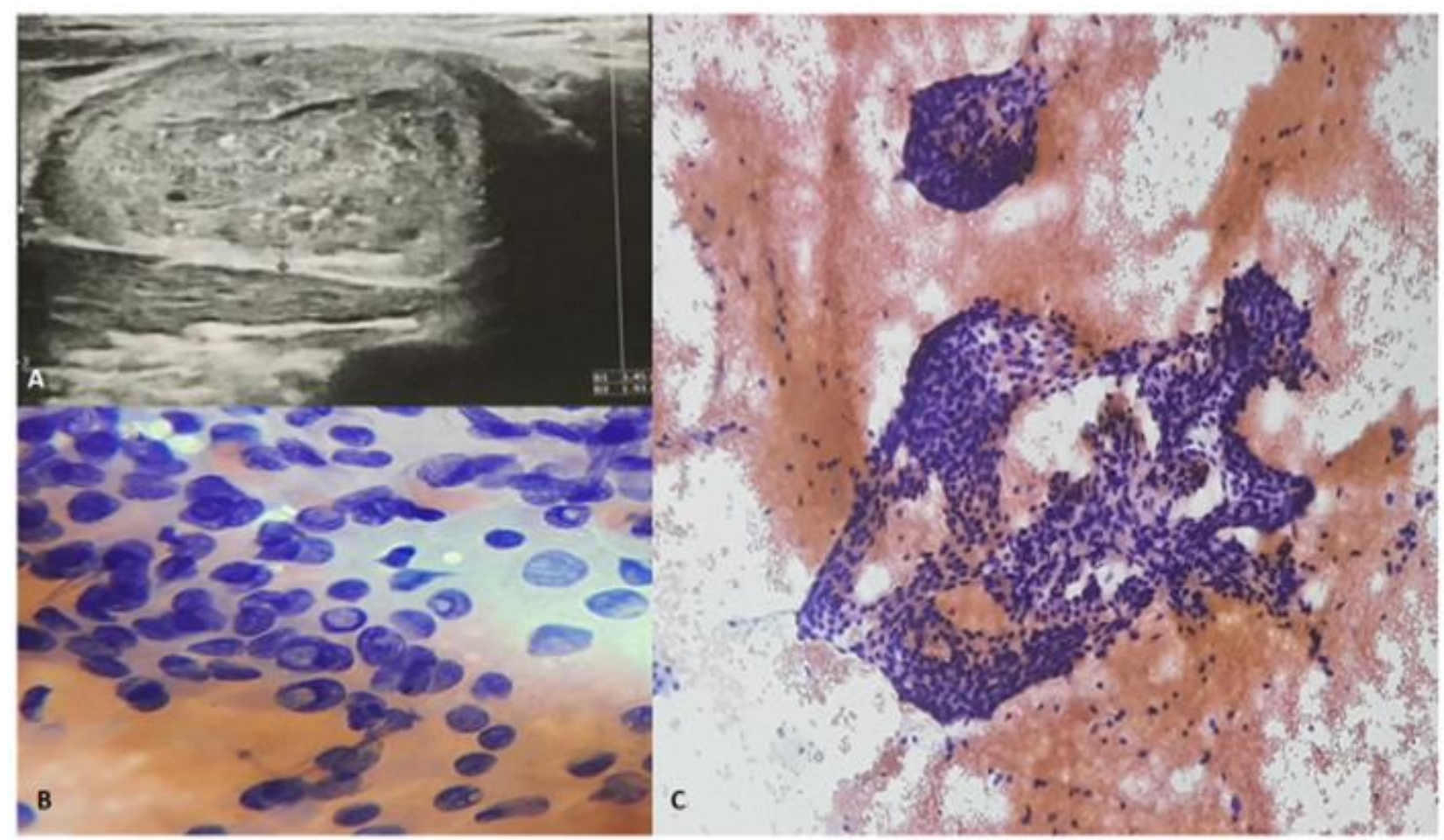

A) Ultrasonography- hyperechoic right lobe thyroid with multiple punctate echogenic foci. B \& C) FNAC-atypical cells in papillaroid clusters with nuclear enlargement, irregular nuclear border, intranuclear cytoplasmic inclusions

A total thyroidectomy with central compartment lymph node dissection and modified radical neck dissection was performed. The specimens were submitted for pathological examination. Right lobe of thyroid measures $4.5 \times 2 \times 2 \mathrm{~cm}$, left lobe with isthmus measures $4.5 \times 3 \times 2 \mathrm{~cm}$. Surface of thyroid was unremarkable. Cutting through superior pole-cut section showed multiple grey white granular lesion (more than 15 foci) largest measuring $1.1 \times 0.7 \times 0.6$ $\mathrm{cm}$ which is at a distance of $0.6 \mathrm{~cm}$ from nearest resected margin. Cut section of left lobe and isthmus showed normal colloid filled appearance without any nodular mass. Histological analysis of right lobe of thyroid showed multifocal infiltrating neoplasm in papillary, micropapillary, focal follicular and solid growth pattern with squamous metaplasia, fibrosis, lymphocytic infiltration with lymphoid follicle formation and numerous psammoma bodies. Neoplastic cells lining papillae showed nuclear crowding, overlapping, clearing and grooving. Capsule of the thyroid is infiltrated by neoplasm in areas. Left lobe of thyroid and isthmus showed colloid filled follicles of varying size and lymphocytic infiltration with follicle formation. A diagnosis of Multifocal Diffuse Sclerosing Variant of papillary Thyroid carcinoma was made. The lymph nodes (pretracheal 5/12 and right neck 4/9) showed extensive metastasis from above neoplasm. 


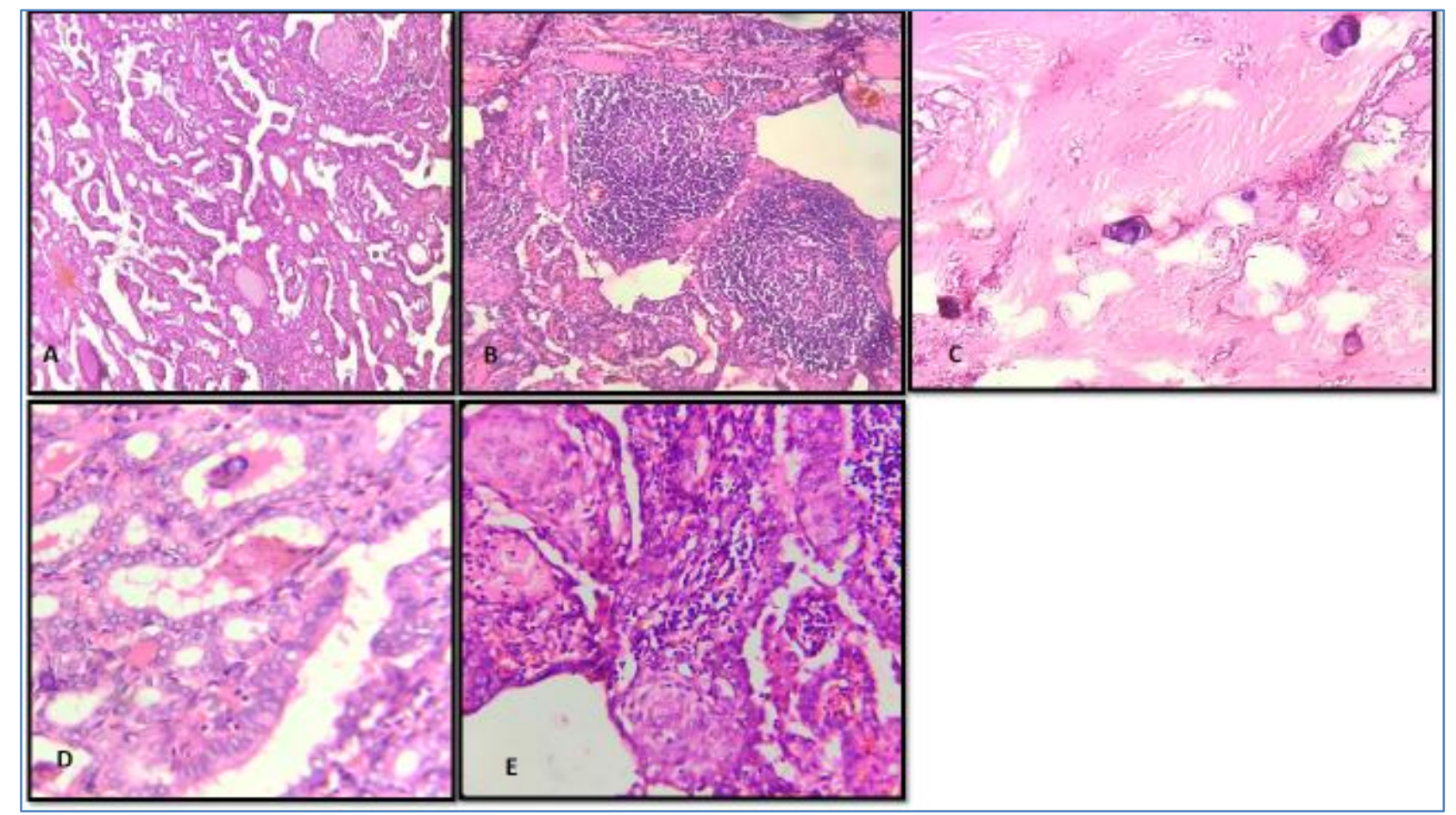

A)Histopathology of DSV PTC showing papillary growth pattern (10x) B) lymphocytic infiltration with follicle formation C)

Dense sclerosis with psammoma bodies. D) Nuclear features like enlargement, crowding, overlapping and optically clear nucleus E) Squamoid nests

\section{DISCUSSION}

We reported the case of an young male presenting as right sided neck swelling in the absence of a visible thyroid swelling who was postoperatively confirmed as Multifocal diffuse sclerosing variant papillary thyroid carcinoma. Although DSV PTC is rare, it is important for clinician to recognize this disease because of its diffuse enlargement and close resemblance to hashimoto's thyroiditis and to avoid a misdiagnosis and mistreatment[4]. In our case thyroid was normal looking prior to surgery with a normal thyroid function and it is only after thyroidectomy that multiple foci of lesions identified. Ultrasound evaluation may be helpful initially because the diagnostic features of DSV PTC include heterogenous echotexture, solid composition, ill-defined margin, and diffuse microcalcification with a snowstrom appearance in thyroid lobes with or without an associated mass [5]. Our case also showed above mentioned heterogenous echotexture with multiple punctate echogenic foci (microcalcifications). The diffuse nature of DSV PTC often mimic chronic thyroiditis on ultrasound image and leads to treatment delay especially in the absence of a nodular mass [6].

Fine needle aspiration cytology of DSV PTC always show numerous psammoma bodies, abundant lymphocytes, metaplastic squamoid cells and epithelial cells that show classic features of papillary thyroid carcinoma such as nuclear grooves, intranuclear inclusion, ground glass nuclei. Pap smear of right neck swelling of our young patient showed atypical epithelial cells arranged in papillaroid pattern with abundant cytoplasm, enlarged nucleus with irregular nuclear membrane, occasional intranuclear inclusion- a metastasis from papillary adenocarcinoma was suspected and a primary in thyroid was likely owing to the presence of intranuclear inclusion. Diagnostic challenge in fine needle aspiration cytology of DSV PTC is due to sampling failure that may occur because of unrepresentative tissue sample obtained from diffuse fibrotic thyroid especially in the absence of significant nodular mass [7].

A total thyroidectomy with lymph node dissection was performed for our patient. Usually a total thyroidectomy results in fewer recurrence. Following total thyroidectomy our patient is on follow up with Thyroglobulin. Thyroglobulin is a sensitive and specific marker for detecting relapses. Often the presence of antithyroglobulin autoantibody in lymphocytic thyroiditis of DSV PTC cases often pose challenges in estimating thyroglobulin level as these antibodies interfere with thyroglobulin assays [8].

In conclusion, as patients with DSV PTC present with diffuse thyroid without any nodularity and mimic hashimoto's thyroiditis a thorough clinical evaluation and early diagnosis are important. DSV PTC has greater tendency for lymph node and distant metastasis and worse disease free survival than other PTC variants 
Ann Maria Sunny \& Sankar S; Saudi J Pathol Microbiol, Jan, 2022; 7(1): 20-23

\section{REFERENCES}

1. Chen, C. C., Chen, W. C., Peng, S. L., \& Huang, S. M. (2013). Diffuse sclerosing variant of thyroid papillary carcinoma: diagnostic challenges occur with Hashimoto's thyroiditis. Journal of the Formosan Medical Association, 112(6), 358-362.

2. Kinoshita, Y., Takasu, K., Yuri, T., Yoshizawa, K., Emoto, Y., Tsubura, A., \& Shikata, N. (2013). Estrogen receptor-and progesterone receptorpositive diffuse sclerosing variant of papillary thyroid carcinoma: a case report. Case reports in oncology, 6(1), 216-223.

3. Jeong, S. H., Hong, H. S., Lee, E. H., \& Kwak, J. J. (2016). The diffuse sclerosing variant of papillary thyroid cancer presenting as innumerable diffuse microcalcifications in underlying adolescent Hashimoto's thyroiditis: a case report. Medicine, 95(12).

4. Lee, J. Y., Shin, J. H., Han, B. K., Ko, E. Y., Kang, S. S., Kim, J. Y., ... \& Chung, J. H. (2007). Diffuse sclerosing variant of papillary carcinoma of the thyroid: imaging and cytologic

findings. Thyroid, 17(6), 567-573.

5. Joung, J. Y., Kim, T. H., Jeong, D. J., Park, S. M., Cho, Y. Y., Jang, H. W., ... \& Kim, S. W. (2016). Diffuse sclerosing variant of papillary thyroid carcinoma: major genetic alterations and prognostic implications. Histopathology, 69(1), 4553.

6. Koo, J. S., Hong, S., \& Park, C. S. (2009). Diffuse sclerosing variant is a major subtype of papillary thyroid carcinoma in the young. Thyroid, 19(11), 1225-1231.

7. Chow, S. M., Chan, J. K. C., Law, S. C. K., Tang, D. L. C., Ho, C. M., Cheung, W. Y., ... \& Lau, W. H. (2003). Diffuse sclerosing variant of papillary thyroid carcinoma-clinical features and outcome. European Journal of Surgical Oncology (EJSO), 29(5), 446-449.

8. Fujimoto, Y., Obara, T., Ito, Y., Kodama, T., Aiba, M., \& Yamaguchi, K. (1990). Diffuse sclerosing variant of papillary carcinoma of the thyroid: Clinical importance, surgical treatment, and follow-up study. Cancer, 66(11), 2306-2312. 